\title{
Rectangular M-tensors and strong rectangular M-tensors
}

\author{
Jun $\mathrm{He}^{*}$, Yanmin Liu, Guangjun Xu \\ School of Mathematics, Zunyi Normal College, Zunyi, Guizhou 563006 China \\ *Corresponding author, e-mail: hejunfan1@163.com
}

Received 23 Sep 2020

Accepted 23 Feb 2021

ABSTRACT: In this paper, two new classes of rectangular tensors called rectangular M-tensors and strong rectangular M-tensors are introduced. It is shown that an even-order partially symmetric rectangular M-tensor is positive semidefinite and an even-order partially symmetric strong rectangular M-tensor is positive definite. As a generalization of rectangular M-tensors, we introduce the rectangular H-tensors. In addition, some properties of (strong) rectangular M-tensors are established.

KEYWORDS: rectangular tensor, H-rectangular tensor, V-singular value, positive definiteness

MSC2010: 15A18 15A69 65F15

\section{INTRODUCTION}

Let $\mathbb{R}(\mathbb{C})$ be the real (complex) field, $p, q, m, n$ be positive integers, $m, n \geqslant 2,[n]=\{1,2, \ldots, n\}$. A $(p, q)$-th order $(m \times n)$-dimensional real rectangular tensor, denoted by $\mathscr{A}=\left(a_{i_{1} \ldots i_{p} j_{1} \ldots j_{q}}\right) \in \mathbb{R}^{[p ; q ; m ; n]}$, is defined as follows:

$$
a_{i_{1} \ldots i_{p} j_{1} \ldots j_{q}} \in \mathbb{R}, \quad i_{1}, \ldots, i_{p} \in[m], \quad j_{1}, \ldots, j_{q} \in[n]
$$

$\mathscr{A}$ is called nonnegative if $a_{i_{1} \ldots i_{p} j_{1} \ldots j_{q}} \geqslant 0$, denoted by $\mathscr{A} \in \mathbb{R}_{+}^{[p ; q ; m ; n]}$. A rectangular tensor $\mathscr{A}$ is called real partially symmetric, if $a_{i_{1} \ldots i_{p} j_{1} \ldots j_{q}}$ is invariant under any permutation of indices among $i_{1} \ldots i_{p}$, and any permutation of indices among $j_{1} \ldots j_{q}$, i.e.,

$$
a_{\pi\left(i_{1} \ldots i_{p}\right) \sigma\left(j_{1} \ldots j_{q}\right)}=a_{i_{1} \ldots i_{p} j_{1} \ldots j_{q}}, \quad \pi \in S_{p}, \quad \sigma \in S_{q},
$$

where $S_{r}$ is the permutation group of $r$ indices. When $p, q$ are even, $\mathscr{A}$ is called even-order partially symmetric.

For any vectors $x \in \mathbb{C}^{m}, y \in \mathbb{C}^{n}$, let $\mathscr{A} x^{p-1} y^{q}$ be a vector in $\mathbb{C}^{m}$ such that

$$
\begin{aligned}
& \left(\mathscr{A} x^{p-1} y^{q}\right)_{i}= \\
& \sum_{i_{2}, \ldots, i_{p}=1}^{m} \sum_{j_{1}, \ldots, j_{q}=1}^{n} a_{i i_{2} \ldots i_{p} j_{1} \ldots j_{q}} x_{i_{2}} \ldots x_{i_{p}} y_{j_{1}} \ldots y_{j_{q}},
\end{aligned}
$$

where $i \in[m]$. Let $\mathscr{A} x^{p} y^{q-1}$ be a vector in $\mathbb{C}^{n}$ such that

$$
\begin{aligned}
& \left(\mathscr{A} x^{p} y^{q-1}\right)_{j}= \\
& \sum_{i_{1}, \ldots, i_{p}=1}^{m} \sum_{j_{2}, \ldots, j_{q}=1}^{n} a_{i_{1} i_{2} \ldots i_{p} j j_{2} \ldots j_{q}} x_{i_{1}} \ldots x_{i_{p}} y_{j_{2}} \ldots y_{j_{q}},
\end{aligned}
$$

where $j \in[n]$.

Definition 1 [1] Let $\mathscr{A} \in \mathbb{R}^{[p ; q ; m ; n]}$ be a partially symmetric rectangular tensor, if there exist a number $\lambda \in \mathbb{C}$ and the vectors $x \in \mathbb{C}^{m} \backslash\{0\}, y \in \mathbb{C}^{n} \backslash\{0\}$ such that

$$
\mathscr{A} x^{p-1} y^{q}=\lambda x^{[l-1]}, \quad \mathscr{A} x^{p} y^{q-1}=\lambda y^{[l-1]},
$$

where $x^{[\alpha]}=\left[x_{1}^{\alpha}, \ldots, x_{n}^{\alpha}\right]^{\mathrm{T}}$ and $l=p+q$, then $\lambda$ is called the singular value of $\mathscr{A}$, and $(x, y)$ is the left and right eigenvectors pair of $\mathscr{A}$, associated with $\lambda$. If $\lambda \in \mathbb{R}, x \in \mathbb{R}^{m}$, and $y \in \mathbb{R}^{n}$, then $\lambda$ is called the $H$-singular value of $\mathscr{A}$, and $(x, y)$ is the left and right $H$-eigenvectors pair associated with $\lambda$.

In order to verify the positive definiteness of a $(p, q)$-th order $(m \times n)$-dimensional partially symmetric rectangular tensor, the definition of V-singular value is introduced as follows.

Definition 2 [2] Let $\mathscr{A} \in \mathbb{R}^{[p ; q ; m ; n]}$ be a partially symmetric rectangular tensor, $p, q \geqslant 2$. If there exist a number $\lambda \in \mathbb{R}$, vectors $x \in \mathbb{R}^{m} \backslash\{0\}$, and 
$y \in \mathbb{R}^{n} \backslash\{0\}$ such that

$$
\begin{gathered}
\mathscr{A} x^{p-1} y^{q}=\lambda x^{[p-1]}, \quad \mathscr{A} x^{p} y^{q-1}=\lambda y^{[q-1]}, \\
\sum_{i=1}^{m} x_{i}^{p}=1, \quad \sum_{j=1}^{n} y_{j}^{q}=1,
\end{gathered}
$$

then $\lambda$ is called the V-singular value of $\mathscr{A}$, and $(x, y)$ is the left and right eigenvectors pair of $\mathscr{A}$, associated with $\lambda$.

Suppose that $\mathscr{A} \in \mathbb{R}^{[p ; q ; m ; n]}$ is a partially symmetric rectangular tensor, $p$ and $q$ are even. Then,

$$
\begin{aligned}
& f(x, y)=\mathscr{A} x^{p} y^{q}= \\
& \sum_{i_{1}, \ldots, i_{p}=1}^{m} \sum_{j_{1}, \ldots, j_{q}=1}^{n} a_{i_{1} \ldots i_{p} j_{1} \ldots j_{q}} x_{i_{1}} \ldots x_{i_{p}} y_{j_{1}} \ldots y_{j_{q}}>0
\end{aligned}
$$

for all nonzero vectors $x \in \mathbb{R}^{m}, y \in \mathbb{R}^{n}$ if and only if $\mathscr{A}$ is positive definite. $\mathscr{A}$ is called an elasticity tensor, if $p=q=2, m=n=2$ or 3 , and $\mathscr{A}$ is a real partially symmetric rectangular tensor. When $\mathscr{A}$ is an elasticity tensor, the strong ellipticity condition holds if and only if $\mathscr{A}$ is positive definite, the strong ellipticity condition plays an important role in the theory of elasticity [3-5]. The following necessary and sufficient conditions for the positive definiteness of a partially symmetric rectangular tensor are provided in as follows.

Theorem $1([\mathbf{1}, \mathbf{2}])$ Suppose that $\mathscr{A} \in \mathbb{R}^{[p ; q ; m ; n]}$ is a partially symmetric rectangular tensor, $p$ and $q$ are even. Then,

(a) $\mathscr{A}$ is positive definite if and only if all of its $H$-singular values are positive.

(b) $\mathscr{A}$ is positive definite if and only if all of its $V$-singular values are positive.

Eigenvalue problems of square tensor have been drew widespread attention [6-8]. In order to verify the positive definiteness of an $m$ th-order $n$ dimensional real square symmetric tensor $\mathscr{A}$, the definition of H-eigenvalue is introduced by Qi in [9].

Definition 3 [9] Let $\mathscr{A} \in \mathbb{R}^{[m, n]}$ be an $m$ th-order $n$-dimensional real square tensor, if there exists a vector $x \in \mathbb{R}^{n}$ and a number $\lambda \in \mathbb{R}$ such that

$$
\mathscr{A} x^{m-1}=\lambda x^{[m-1]}
$$

where

$$
\begin{aligned}
\mathscr{A} x^{m-1} & =\left(\sum_{i_{2}, \cdots, i_{m}=1}^{n} a_{i i_{2} \ldots i_{m}} x_{i_{2}} \ldots x_{i_{m}}\right)_{i \in[n]}, \\
x^{[m-1]} & =\left(x_{i}^{m-1}\right)_{i \in[n]},
\end{aligned}
$$

then $\lambda$ is called an $\mathrm{H}$-eigenvalue of $\mathscr{A}$ and $x$ is called an $\mathrm{H}$-eigenvector of $\mathscr{A}$ associated with $\lambda$.

Recently, M-tensors and strong M-tensors are introduced as the generalizations of the well-known M-matrices [10-12], the authors proved that an even-order symmetric M-tensor is positive semidefinite and an even-order symmetric strong M-tensor is positive definite [12-14].

Definition $4[10,12]$ A tensor $\mathscr{A} \in \mathbb{R}^{[m, n]}$ is called an M-tensor, if there exist a nonnegative tensor $\mathscr{B}$ and a positive real number $s \geqslant \rho(\mathscr{B})$ where

$$
\rho_{H}(\mathscr{A})=\max \{|\lambda|: \lambda \text { is an H-eigenvalue of } \mathscr{A}\}
$$

such that

$$
\mathscr{A}=s \mathscr{I}-\mathscr{B},
$$

in which $\mathscr{I}=\left(\delta_{i_{1} \ldots i_{m}}\right)$ is the $m$ th order $n$ dimensional identity tensor with

$$
\delta_{i_{1} \ldots i_{m}}= \begin{cases}1, & \text { if } i_{1}=\ldots=i_{m} \\ 0, & \text { otherwise }\end{cases}
$$

Furthermore, if $s>\rho_{H}(\mathscr{B})$, then $A$ is called a strong M-tensor.

Lately, Ding et al [5] introduced a structured partially symmetric tensor named elasticity M-tensors, and proved that a nonsingular elasticity M-tensor is positive definite.

Definition 5 [5] Let $\mathscr{A} \in \mathbb{R}^{[2 ; 2 ; m ; n]}, x=\left(x_{i}\right)_{i=1}^{m} \in$ $\mathbb{R}^{m} \backslash\{0\}, y=\left(y_{l}\right)_{l=1}^{n} \in \mathbb{R}^{n} \backslash\{0\}$ and $\lambda \in \mathbb{R}$, such that

$$
\left\{\begin{array}{l}
\mathscr{A} x y y=\lambda x, \\
\mathscr{A} x x y=\lambda y, \\
x^{\mathrm{T}} x=1, y^{\mathrm{T}} y=1,
\end{array}\right.
$$

where

$$
\begin{aligned}
& (\mathscr{A} x y y)_{i}=\sum a_{i j k l} x_{j} y_{k} y_{l}, \\
& (\mathscr{A} x x y)_{l}=\sum a_{i j k l} x_{i} x_{j} y_{k} .
\end{aligned}
$$

Then $\lambda$ is called an M-eigenvalue of $\mathscr{A}$, the vectors $x$ and $y$ are called the corresponding M-eigenvectors.

Definition 6 [5] A partially symmetric tensor $\mathscr{A} \in$ $\mathbb{R}^{[2 ; 2 ; n ; n]}$ is called an elasticity M-tensor if there exist a nonnegative partially symmetric tensor $\mathscr{B} \in$ $\mathbb{R}^{[2 ; 2 ; n ; n]}$ and a real number $s \geqslant \rho_{M}(\mathscr{B})$, where

$$
\rho_{M}(\mathscr{A})=\max \{|\lambda|: \lambda \text { is an M-eigenvalue of } \mathscr{A}\}
$$

such that

$$
\mathscr{A}=s \mathscr{I}_{E}-\mathscr{B}
$$


in which $\mathscr{I}_{E}=\left(e_{i j k l}\right) \in \mathbb{R}^{[2 ; 2 ; n ; n]}$ is the elasticity identity tensor with

$$
e_{i j k l}= \begin{cases}1, & \text { if } i=j \text { and } k=l, \\ 0, & \text { otherwise. }\end{cases}
$$

Furthermore, if $s>\rho_{M}(\mathscr{B})$, then $\mathscr{A}$ is called a nonsingular elasticity M-tensor.

\section{NOTATION AND PRELIMINARIES}

In this section, we shall introduce some definitions and important properties related to eigenvalue of a tensor, which are needed in the subsequent analysis.

Let $\mathbb{R}_{+}^{n}$ denote the cone of nonnegative vectors. We use small letters $a, b, \ldots$ for scalars, small letters $x, y, \ldots$ for vectors, capital letters $A, B, \ldots$ for matrices, calligraphic letters $\mathscr{A}, \mathscr{B}, \ldots$ for tensors. The $i$-th entry of a vector $x$ is denoted by $x_{i}$, the $(i, j)$-th entry of a matrix $A$ is denoted by $a_{i j}$ and the $\left(i_{1} \ldots i_{p} j_{1} \ldots j_{q}\right)$-th entry of a rectangular tensor $\mathscr{A}$ is denoted by $a_{i_{1} \ldots i_{p} j_{1} \ldots j_{q}}$. For any rectangular tensor $\mathscr{A}=\left(a_{i_{1} \ldots i_{p} j_{1} \ldots j_{q}}\right) \in \mathbb{R}^{[p ; q ; m ; n]}$, denote $|\mathscr{A}|=$ $\left(\left|a_{i_{1} \ldots i_{p} j_{1} \ldots j_{q}}\right|\right)$.

The Perron-Frobenius theorem for nonnegative square tensors is introduced in [15], which states that the spectral radius of any nonnegative square tensor is an eigenvalue with a nonnegative eigenvector. Denote $\lambda_{\max }(\mathscr{A})$ as the maximal V-singular value of $\mathscr{A} \in \mathbb{R}^{[p ; q ; m ; n]}$. Then

$\lambda_{\max }(\mathscr{A})=\max \left\{\mathscr{A} x^{p} y^{q}, \sum_{i=1}^{m} x_{i}^{p}=1, \sum_{j=1}^{n} y_{j}^{q}=1\right\}$.

Let $\sigma(\mathscr{A})$ be the set containing all V-singular values of $\mathscr{A}=\left(a_{i_{1} \ldots i_{p} j_{1} \ldots j_{q}}\right) \in \mathbb{R}^{[p ; q ; m ; n]}$. And we call

$$
\rho_{V}(\mathscr{A})=\max \{|\lambda|: \lambda \in \sigma(\mathscr{A})\}
$$

is the largest $\mathrm{V}$-singular value of $\mathscr{A}$. The following Perron-Frobenius theorem for $\mathrm{V}$-singular values of nonnegative square rectangular tensors is introduced in [2].

Lemma 1 Let $\mathscr{A}=\left(a_{i_{1} \ldots i_{p} j_{1} \ldots j_{q}}\right) \in \mathbb{R}_{+}^{[p ; q ; m ; n]}$. Then, $\rho_{V}(\mathscr{A})=\lambda_{\max }(\mathscr{A})$, and there is a pair of nonnegative eigenvectors corresponding to the $\rho_{V}(\mathscr{A})$.

\section{RECTANGULAR M-TENSORS AND STRONG RECTANGULAR M-TENSORS}

In this section, we firstly introduce (strong) rectangular M-tensors which are different from (strong) M-tensors. Moreover, the positive definiteness of an even-order partially symmetric strong M-tensor is proved. The identity tensor $\mathscr{I}$ plays an important role in the definition of $\mathrm{M}$-tensor, because

$$
\mathscr{I} x^{m-1}=x^{[m-1]}
$$

always holds for any nonzero vector $x$. That is to say, 1 is an unique $\mathrm{H}$-eigenvalue of the identity tensor $\mathscr{I}$. Similarly, the elasticity identity tensor $\mathscr{I}_{E}$ also plays an important role in the definition of elasticity M-tensor, because

$$
\mathscr{I}_{E} x y^{2}=x, \mathscr{I}_{E} x^{2} y=y
$$

always holds for any nonzero vectors $x, y$. That is to say, 1 is an unique M-eigenvalue of the identity tensor $\mathscr{I}$. We next introduce (strong) rectangular M-tensors based on the so-called rectangular identity tensor.

Definition 7 A tensor $\mathscr{A} \in \mathbb{R}^{[p ; q ; m ; n]}$ is called a rectangular M-tensor if there exist a partially symmetric nonnegative tensor $\mathscr{B} \in \mathbb{R}^{[p ; q ; m ; n]}$ and a real number $s \geqslant \rho_{V}(\mathscr{B})$, where

$\rho_{V}(\mathscr{B})=\max \{|\lambda|: \lambda$ is a V-singular value of $\mathscr{B}\}$,

such that

$$
\mathscr{A}=s \mathscr{I}_{R}-\mathscr{B},
$$

in which $\mathscr{I}_{R}=\left(\epsilon_{i_{1} \ldots i_{p} j_{1} \ldots j_{q}}\right) \in \mathbb{R}^{[p ; q ; n ; n]}$ is the rectangular identity tensor with

$$
\epsilon_{i_{1} \ldots i_{p} j_{1} \ldots j_{q}}= \begin{cases}1, & \text { if } i_{1}=\cdots=i_{p} \text { and } j_{1}=\cdots=j_{q}, \\ 0, & \text { otherwise. }\end{cases}
$$

Furthermore, if $s>\rho_{V}(\mathscr{B})$, then $\mathscr{A}$ is called a strong rectangular M-tensor.

Remark 1 If $\mathscr{A} \in \mathbb{R}^{[p ; q ; m ; n]}$ is a partially symmetric rectangular tensor, $p$ and $q$ are even. Then, there exist V-singular value of $\mathscr{A}$ and associated left and right eigenvectors [2].

Remark 2 From the definition of rectangular identity tensor, we can get, 1 is its unique V-singular value of $\mathscr{I}_{R}$.

Remark 3 If $\mathscr{A} \in \mathbb{R}^{[2 ; 2 ; n ; n]}$ and $\mathscr{B} \in \mathbb{R}^{[2 ; 2 ; n ; n]}$ are partially symmetric, then, the definition of the rectangular M-tensor is the same as the definition of the elasticity M-tensor. Therefore, the rectangular M-tensor can be regarded as a generalization of the elasticity M-tensor. 
Theorem 2 Let $\mathscr{B} \in \mathbb{R}^{[p ; q ; m ; n]}$ be a partially symmetric rectangular tensor, $\mathscr{A}=a\left(\mathscr{B}+b \mathscr{I}_{R}\right)$, where a and $b$ are two real numbers. Then $\mu$ is a V-singular value of $\mathscr{A}$ if and only if $\mu=a(\lambda+b)$ and $\lambda$ is $a$ $V$-singular value of $\mathscr{B}$. In this case, they have the same eigenvectors pair.

Proof: If $\lambda$ is a V-singular value of $\mathscr{B}$ with eigenvectors pair $(x, y)$, then

$$
\begin{gathered}
\mathscr{B} x^{p-1} y^{q}=\lambda x^{[p-1]}, \quad \mathscr{B} x^{p} y^{q-1}=\lambda y^{[q-1]}, \\
\sum_{i=1}^{m} x_{i}^{p}=1, \quad \sum_{j=1}^{n} y_{j}^{q}=1 .
\end{gathered}
$$

Since $\mathscr{I}_{R}$ is a rectangular identity tensor, then

$$
\mathscr{I}_{R} x^{p-1} y^{q}=x^{[p-1]}, \quad \mathscr{I}_{R} x^{p} y^{q-1}=y^{[q-1]}
$$

From (5) and (6), we have

$$
\begin{aligned}
& a\left(\mathscr{B}+b \mathscr{I}_{R}\right) x^{p-1} y^{q}=a(\lambda+b) x^{[p-1]}, \\
& a\left(\mathscr{B}+b \mathscr{I}_{R}\right) x^{p} y^{q-1}=a(\lambda+b) y^{[q-1]},
\end{aligned}
$$

which means

$$
\mathscr{A} x^{p-1} y^{q}=\mu x^{[p-1]}, \quad \mathscr{A} x^{p} y^{q-1}=\mu y^{[q-1]},
$$

i.e., $\mu$ is a V-singular value of $\mathscr{A}$ with eigenvectors pair $(x, y)$.

On the other side, if $a=0$, the result is trivial. If $a \neq 0$, suppose $\mu$ is a V-singular value of $\mathscr{A}$ with eigenvectors pair $(x, y)$, then

$$
\begin{aligned}
\mathscr{B} x^{p-1} y^{q} & =\frac{1}{a}(\mu-a b) x^{[p-1]}, \\
\mathscr{B} x^{p} y^{q-1} & =\frac{1}{a}(\mu-a b) y^{[q-1]},
\end{aligned}
$$

i.e., $\lambda=\frac{1}{a}(\mu-a b)$ is a V-singular value of $\mathscr{B}$ with eigenvectors pair $(x, y)$.

Corollary 1 Suppose $\mathscr{B} \in \mathbb{R}^{[p ; q ; m ; n]}$ is partially symmetric, $s$ is a real numbers and $\mathscr{A}=s \mathscr{I}_{R}-\mathscr{B}$. Then for any $V$-singular value $\eta$ of $\mathscr{A}$ with eigenvectors pair $(x, y)$, there exists a $V$-singular value $\theta=s-\eta$ of $\mathscr{B}$ with same eigenvectors pair $(x, y)$.

It is showed that all $\mathrm{H}$-eigenvalues of a M-tensor are nonnegative, and all $\mathrm{H}$-eigenvalues of a strong M-tensor are positive $[10,12]$. For rectangular Mtensors and strong rectangular M-tensors, the following spectral properties are presented.

Theorem 3 If $\mathscr{A}=s \mathscr{I}_{R}-\mathscr{B}$ is a partially symmetric rectangular $M$-tensor and $\eta$ is a $V$-singular value of $\mathscr{A}$, then $\eta$ is nonnegative. If $\mathscr{A}=s \mathscr{I}_{R}-\mathscr{B}$ is a partially symmetric strong rectangular M-tensor and $\eta$ is a V-singular value of $\mathscr{A}$, then $\eta$ is positive.
Proof: Let $\rho_{V}(\mathscr{B})$ be the largest V-singular value of $\mathscr{B}$, according to Corollary 1 , there exists a V-singular value $\theta$ of $\mathscr{B}$ such that

$$
\eta=s-\theta .
$$

Since $\mathscr{A}=s \mathscr{I}_{R}-\mathscr{B}$ is a rectangular M-tensor, then

$$
\eta=s-\theta \geqslant s-\rho_{V}(\mathscr{B}) .
$$

Similarly, the results about strong rectangular M-tensors can be obtained.

Theorem 4 Let a partially symmetric rectangular tensor $\mathscr{B}$ be nonnegative, irreducible and $\mathscr{A}=s \mathscr{I}_{R}-$ $\mathscr{B}$ be a rectangular $M$-tensor. Then the smallest $V$-singular value of $\mathscr{A}$ is nonnegative and its corresponding eigenvectors are positive. If $\mathscr{A}$ is a strong rectangular $M$-tensor, then the smallest V-singular value of $\mathscr{A}$ is positive and its corresponding eigenvectors are positive.

Proof: By Lemma 1, we know that $\rho_{V}(\mathscr{B})$ is a positive V-singular value with positive eigenvectors. By Corollary 1 , we can see that $c=s-\rho_{V}(\mathscr{B}) \geqslant 0$ is a V-singular value of $\mathscr{A}$ and they have the same eigenvectors. If $\mathscr{A}$ is a strong rectangular M-tensor, note that $c=s-\rho_{V}(\mathscr{B})>0$.

The entries $a_{i \ldots i j \ldots j}(i \in[m], j \in[n])$ are called diagonal, and other entries are called off-diagonal. A rectangular tensor in $\mathbb{R}^{[p ; q ; m ; n]}$ is called a rectangular Z-tensor if all its off-diagonal entries are nonpositive.

Theorem 5 Let $\mathscr{A} \in \mathbb{R}^{[p ; q ; m ; n]}$ be a partially symmetric rectangular $Z$-tensor. Then $\mathscr{A}$ is a strong rectangular $M$-tensor if and only if $\alpha>\rho_{V}\left(\alpha \mathscr{I}_{R}-\mathscr{A}\right)$, where $\alpha=\max _{i \in[m], j \in[n]}\left\{a_{i \ldots i j \ldots j}\right\}$.

Proof: If $\alpha>\rho_{V}\left(\alpha \mathscr{I}_{R}-\mathscr{A}\right)$, by $\mathscr{A}=\alpha \mathscr{I}_{R}-\left(\alpha \mathscr{I}_{R}-\mathscr{A}\right)$ and the definition of strong rectangular M-tensors, then $\mathscr{A}$ is a strong rectangular M-tensor.

If $\mathscr{A}$ is a strong rectangular M-tensor, then $\mathscr{A}$ can be written as $\mathscr{A}=s \mathscr{I}_{R}-\mathscr{B}$, where $\mathscr{B}$ is a nonnegative rectangular tensor and $s>\rho_{V}(\mathscr{B})$. Then $\alpha \mathscr{I}_{R}-\mathscr{A}=(\alpha-s) \mathscr{I}_{R}+\mathscr{B}$, which yields $\alpha-$ $\rho_{v}\left(\alpha \mathscr{I}_{R}-\mathscr{A}\right)=\rho_{v}\left(s \mathscr{I}_{R}-\mathscr{B}\right)>0$, therefore $\alpha>$ $\rho_{v}\left(\alpha \mathscr{I}_{R}-\mathscr{A}\right)$.

Theorem $6 \mathscr{A} \in \mathbb{R}^{[p ; q ; m ; n]}$ is a rectangular M-tensor if and only if $\mathscr{A}+t \mathscr{I}_{R}$ is a strong rectangular $M$-tensor for any $t>0$.

Proof: If $\mathscr{A}+t \mathscr{I}_{R}$ is a strong rectangular M-tensor for any $t>0$, when $t$ approaches 0 , then $\mathscr{A}$ is a strong rectangular M-tensor. 
If $\mathscr{A}$ is a strong rectangular M-tensor, then $\mathscr{A}$ can be written as $\mathscr{A}=s \mathscr{I}_{R}-\mathscr{B}$, where $\mathscr{B}$ is a nonnegative rectangular tensor and $s>\rho_{V}(\mathscr{B})$. Then $\mathscr{A}+t \mathscr{I}_{R}=(s+t) \mathscr{I}_{R}-\mathscr{B}$, which yields that, $\mathscr{A}+t \mathscr{I}_{R}$ is a strong rectangular M-tensor.

Theorem 7 When $p, q$ are even, let $\mathscr{A} \in \mathbb{R}^{[p ; q ; m ; n]}$ be a partially symmetric rectangular Z-tensor. Then $\mathscr{A}$ is a strong rectangular $M$-tensor if and only if $\mathscr{A}$ is positive definite, and $\mathscr{A}$ is a rectangular $M$-tensor if and only if $\mathscr{A}$ is positive semidefinite.

Proof: When $p, q$ are even, if $\mathscr{A} \in \mathbb{R}^{[p ; q ; m ; n]}$ is a strong rectangular M-tensor, by Theorem 3 and Theorem 4 , then $\mathscr{A}$ is positive definite.

If $\mathscr{A}$ is positive definite, then for any vectors $x, y \neq 0, \mathscr{A} x^{p} y^{q}>0$. Denote $\mathscr{A}=s \mathscr{I}_{R}-\mathscr{B}$, where $\mathscr{B}$ is a nonnegative rectangular tensor, then $\left(s \mathscr{I}_{R}-\right.$ $\mathscr{B}) x^{p} y^{q}>0$, which yields $s>\rho_{V}(\mathscr{B})$ by $\sum_{i=1}^{m} x_{i}^{p}=$ 1, $\sum_{j=1}^{n} y_{j}^{q}=1$. The result for rectangular M-tensors can be obtained similarly.

Let $\mathscr{A} x^{p} \in \mathbb{R}^{[q, n]}$ be a real $q$ th-order $n$ dimensional square tensor, $\mathscr{A} y^{q} \in \mathbb{R}^{[p, m]}$ be a real $p$ th-order $m$-dimensional square tensor, where

$$
\begin{aligned}
& \left(\mathscr{A} x^{p}\right)_{j_{1} \ldots j_{q}}=\sum_{j_{1}, \ldots, j_{q}=1}^{n} a_{i_{1} i_{2} \ldots i_{p} j_{1} \ldots j_{q}} x_{i_{1}} \ldots x_{i_{p}}, \\
& \left(\mathscr{A} y^{p}\right)_{i_{1} \ldots i_{p}}=\sum_{i_{1}, \ldots, i_{p}=1}^{m} a_{i_{1} i_{2} \ldots i_{p} j_{1} \ldots j_{q}} y_{j_{1}} \ldots y_{j_{q}} .
\end{aligned}
$$

The following propositions can be obtained from the definitions of $\mathscr{A} x^{p}$ and $\mathscr{A} y^{q}$.

Theorem 8 When $p, q$ are even, $\mathscr{A} \in \mathbb{R}^{[p ; q ; m ; n]}$ is a partially symmetric rectangular Z-tensor. Then $\mathscr{A}$ is a strong rectangular $M$-tensor if and only if $\mathscr{A} x^{p}$ is a strong $M$-tensor for each $x \geqslant 0, \mathscr{A}$ is a rectangular $M$-tensor if and only if $\mathscr{A} x^{p}$ is a $M$-tensor for each $x \geqslant 0$.

Proof: When $p, q$ are even, if $\mathscr{A} \in \mathbb{R}^{[p ; q ; m ; n]}$ is a strong rectangular M-tensor, by Theorem 3 , then for any vectors $y \neq 0, \mathscr{A} x^{p} y^{q}>0$, which yields $\mathscr{A} x^{p}$ is positive definite. And we find that, $\mathscr{A} x^{p}$ is a Z-tensor for each $x \geqslant 0$. Therefore, $\mathscr{A} x^{p}$ is a strong M-tensor.

If $\mathscr{A} x^{p}$ is a strong M-tensor, then for any vectors $y \neq 0, \mathscr{A} x^{p} y^{q}>0$. Denote $\mathscr{A}=s \mathscr{I}_{R}-\mathscr{B}$, where $\mathscr{B}$ is a nonnegative rectangular tensor, then $s>$ $\mathscr{B} x^{p} y^{q}$ for each $x, y \geqslant 0$, which yields $s>\rho_{V}(\mathscr{B})$ by $\sum_{i=1}^{m} x_{i}^{p}=1, \sum_{j=1}^{n} y_{j}^{q}=1$ and Lemma 1 . Therefore, $\mathscr{A}$ is a strong rectangular M-tensor. The result for rectangular M-tensors can be obtained similarly.

Theorem 9 When $p, q$ are even, $\mathscr{A} \in \mathbb{R}^{[p ; q ; m ; n]}$ is a partially symmetric rectangular Z-tensor. Then $\mathscr{A}$ is a strong rectangular M-tensor if and only if $\mathscr{A} y^{q}$ is a strong $M$-tensor for each $y \geqslant 0, \mathscr{A}$ is a rectangular $M$-tensor if and only if $\mathscr{A} y^{q}$ is a $M$-tensor for each $y \geqslant 0$.

The following theorem can be obtained by Theorem 8 and Theorem 9.

Theorem 10 When $p, q$ are even, $\mathscr{A} \in \mathbb{R}^{[p ; q ; m ; n]}$ is a partially symmetric rectangular Z-tensor. Then $\mathscr{A}$ is a strong rectangular $M$-tensor if and only if one of the following conditions satisfies:

(1) For each $x \geqslant 0$, there exists $y \geqslant 0$ such that $\mathscr{A} x^{p} y^{q-1}>0$

(2) For each $x \geqslant 0$, there exists $y>0$ such that $\mathscr{A} x^{p} y^{q-1}>0$

(3) For each $y \geqslant 0$, there exists $x \geqslant 0$ such that $\mathscr{A} x^{p-1} y^{q}>0$

(4) For each $y \geqslant 0$, there exists $x>0$ such that $\mathscr{A} x^{p-1} y^{q}>0$.

\section{RECTANGULAR H-TENSOR AND STRONG RECTANGULAR H-TENSOR}

$\mathscr{A} \in \mathbb{R}^{[p ; q ; m ; n]}$ is a copositive rectangular tensor, if for any $x \in \mathbb{R}_{+}^{m}, y \in \mathbb{R}_{+}^{n}, \mathscr{A} x^{p} y^{q} \geqslant 0, \mathscr{A} \in \mathbb{R}^{[p ; q ; m ; n]}$ is a strictly copositive rectangular tensor, if for any $0 \neq x \in \mathbb{R}_{+}^{m}, 0 \neq y \in \mathbb{R}_{+}^{n}, \mathscr{A} x^{p} y^{q}>0$ [16]. The definition of $\mathrm{H}$-tensor was introduced in [10]. In this section, we extend rectangular M-tensors to rectangular $\mathrm{H}$-tensors as follows.

Definition 8 Let $\mathscr{A} \in \mathbb{R}^{[p ; q ; m ; n]}$ be a partially symmetric rectangular tensor. Then $\mathscr{M}(\mathscr{A})=$ $\left(m_{i_{1} \ldots i_{p} j_{1} \ldots j_{q}}\right) \in \mathbb{R}^{[p ; q ; m ; n]}$ is called comparison rectangular tensor of $\mathscr{A}$, whose entries are defined as:

$$
\begin{aligned}
& m_{i_{1} \ldots i_{p} j_{1} \ldots j_{q}} \\
& \quad= \begin{cases}+\left|a_{i_{1} \ldots i_{p} j_{1} \ldots j_{q}}\right|, & \text { if } i_{1}=\cdots=i_{p}, j_{1}=\cdots=j_{q}, \\
-\left|a_{i_{1} \ldots i_{p} j_{1} \ldots j_{q}}\right|, & \text { otherwise. }\end{cases}
\end{aligned}
$$

A rectangular tensor is called a rectangular $\mathrm{H}$-tensor, if its comparison tensor is a rectangular M-tensor, and a rectangular tensor is called a strong rectangular $\mathrm{H}$-tensor, if its comparison tensor is a strong rectangular M-tensor. 
Theorem 11 ([16]) Let $\mathscr{A} \in \mathbb{R}^{[p ; q ; m ; n]}$ be a partially symmetric rectangular tensor. Then $\mathscr{A}$ is copositive if and only if

$$
\begin{array}{r}
N_{\text {min }}^{1}(\mathscr{A}) \equiv \min \left\{\mathscr{A} x^{p} y^{q}: x \in \mathbb{R}_{+}^{m}, y \in \mathbb{R}_{+}^{n},\right. \\
\left.\sum_{i=1}^{m} x_{i}^{l}=1, \sum_{j=1}^{n} y_{j}^{l}=1\right\} \geqslant 0 .
\end{array}
$$

$\mathscr{A}$ is strictly copositive if and only if

$$
\begin{array}{r}
N_{\min }^{1}(\mathscr{A}) \equiv \min \left\{\mathscr{A} x^{p} y^{q}: x \in \mathbb{R}_{+}^{m}, y \in \mathbb{R}_{+}^{n},\right. \\
\left.\sum_{i=1}^{m} x_{i}^{l}=1, \sum_{j=1}^{n} y_{j}^{l}=1\right\}>0 .
\end{array}
$$

A general case of above theorem is given as follows.

Theorem 12 Let $\mathscr{A} \in \mathbb{R}^{[p ; q ; m ; n]}$ be a partially symmetric rectangular tensor. Then $\mathscr{A}$ is copositive if and only if

$$
\begin{array}{r}
N_{\min }^{2}(\mathscr{A}) \equiv \min \left\{\mathscr{A} x^{p} y^{q}: x \in \mathbb{R}_{+}^{m}, y \in \mathbb{R}_{+}^{n},\right. \\
\left.\sum_{i=1}^{m} x_{i}^{p}=1, \sum_{j=1}^{n} y_{j}^{q}=1\right\} \geqslant 0 .
\end{array}
$$

$\mathscr{A}$ is strictly copositive if and only if

$$
\begin{array}{r}
N_{\min }^{2}(\mathscr{A}) \equiv \min \left\{\mathscr{A} x^{p} y^{q}: x \in \mathbb{R}_{+}^{m}, y \in \mathbb{R}_{+}^{n},\right. \\
\left.\sum_{i=1}^{m} x_{i}^{p}=1, \sum_{j=1}^{n} y_{j}^{q}=1\right\}>0 .
\end{array}
$$

Proof: For any $0 \neq x \in \mathbb{R}_{+}^{m}, 0 \neq y \in \mathbb{R}_{+}^{n}$, let

$$
\bar{x}=\frac{x}{\left(\sum_{i=1}^{m} x_{i}^{p}\right)^{\frac{1}{p}}}, \quad \bar{y}=\frac{y}{\left(\sum_{j=1}^{n} y_{j}^{q}\right)^{\frac{1}{q}}},
$$

then $\sum_{i=1}^{m} \bar{x}_{i}^{p}=1, \sum_{j=1}^{n} \bar{y}_{j}^{q}=1$, and

$$
\mathscr{A} \bar{x}^{p} \bar{y}^{q}=\frac{\mathscr{A} x^{p} y^{q}}{\sum_{i=1}^{m} x_{i}^{p} \sum_{j=1}^{n} y_{j}^{q}} .
$$

Therefore, $N_{\text {min }}^{1}(\mathscr{A}) \geqslant 0$ if and only if $N_{\text {min }}^{2}(\mathscr{A}) \geqslant 0$. The second conclusion is obtained similarly.

Theorem 13 When $p, q$ are even, then a partially symmetric rectangular $M$-tensor is copositive, and a partially symmetric strong rectangular M-tensor is strictly copositive.
Proof: If $\mathscr{A}$ is a partially symmetric rectangular Mtensor, when $p, q$ are even, from Theorem 7 in [2], we have

$$
\begin{aligned}
N_{\min }^{2}(\mathscr{A}) & \geqslant \lambda_{\min }(\mathscr{A}) \\
= & \min \left\{\mathscr{A} x^{p} y^{q}: \sum_{i=1}^{m} x_{i}^{p}=1, \sum_{j=1}^{n} y_{j}^{q}=1\right\} \geqslant 0,
\end{aligned}
$$

which yields that, $\mathscr{A}$ is copositive. The second conclusion can be obtained similarly.

Theorem 14 When $p, q$ are even, $\mathscr{A}$ is a partially symmetric rectangular $H$-tensor with nonnegative diagonal entries, then $\mathscr{A}$ is positive semidefinite. If $\mathscr{A}$ is a strong partially symmetric rectangular $\mathrm{H}$-tensor with positive diagonal entries, then $\mathscr{A}$ is positive definite.

Proof: Let $\mathscr{A}=D-\mathscr{B}$, where $D$ is the diagonal part of $\mathscr{A}$. Then its comparison tensor $\mathscr{M}(\mathscr{A})=D-$ $|\mathscr{B}|$ is a partially symmetric rectangular M-tensor. By Theorem 12, $\mathscr{M}(\mathscr{A})$ is copositive, which yields

$$
\mathscr{M}(\mathscr{A}) \bar{x}^{p} \bar{y}^{q}=D \bar{x}^{p} \bar{y}^{q}-|\mathscr{B}| \bar{x}^{p} \bar{y}^{q} \geqslant 0,
$$

where $\bar{x} \in \mathbb{R}_{+}^{m}, \bar{y} \in \mathbb{R}_{+}^{n}, \sum_{i=1}^{m} \bar{x}_{i}^{p}=1, \sum_{j=1}^{n} \bar{y}_{j}^{q}=1$. Then

$$
\begin{aligned}
\mathscr{A} x^{p} y^{q} & =D x^{p} y^{q}-\mathscr{B} x^{p} y^{q} \\
& \geqslant D x^{p} y^{q}-|\mathscr{B}||x|^{p}|y|^{q} \geqslant 0,
\end{aligned}
$$

where $x \in \mathbb{R}^{m}, y \in \mathbb{R}^{n}, \sum_{i=1}^{m} x_{i}^{p}=1, \sum_{j=1}^{n} y_{j}^{q}=1$. Therefore, $\mathscr{A}$ is positive semidefinite. The second conclusion can be obtained similarly.

\section{RECTANGULAR TENSOR COMPLEMENTARITY PROBLEMS}

Let $\mathscr{A}=\left(a_{i_{1} i_{2} \ldots i_{p} j_{1} j_{2} \ldots j_{q}}\right) \in \mathbb{R}^{[p ; q ; m ; n]}, q_{m} \in \mathbb{R}^{m}$ and $q_{n} \in \mathbb{R}^{n}$. The rectangular tensor complementarity problem [17], denoted by $\operatorname{RTCP}\left(\mathscr{A}, q_{m}, q_{n}\right)$, is to find vectors $x \in \mathbb{R}^{m}$ and $y \in \mathbb{R}^{n}$ such that

$$
\begin{aligned}
& q_{m}+\mathscr{A} x^{p-1} y^{q} \geqslant 0, x \geqslant 0, x^{\mathrm{T}}\left(q_{m}+\mathscr{A} x^{p-1} y^{q}\right)=0, \\
& q_{n}+\mathscr{A} x^{p} y^{q-1} \geqslant 0, y \geqslant 0, y^{\mathrm{T}}\left(q_{n}+\mathscr{A} x^{p} y^{q-1}\right)=0 .
\end{aligned}
$$

Vectors $x$ and $y$ are said to be feasible if and only if $x$ and $y$ satisfy the following inequalities:

$$
\begin{aligned}
& q_{m}+\mathscr{A} x^{p-1} y^{q} \geqslant 0, \quad x \geqslant 0, \\
& q_{n}+\mathscr{A} x^{p} y^{q-1} \geqslant 0, \quad y \geqslant 0 .
\end{aligned}
$$


A rectangular tensor $\mathscr{A}=\left(a_{i_{1} i_{2} \ldots i_{p} j_{1} j_{2} \ldots j_{q}}\right) \in \mathbb{R}^{[p ; q ; m ; n]}$ is called a rectangular S-tensor if and only if there exists $0<x \in \mathbb{R}^{m}, 0<y \in \mathbb{R}^{n}$ such that

$$
\mathscr{A} x^{p-1} y^{q}>0, \quad \mathscr{A} x^{p} y^{q-1}>0 .
$$

Then, a strong rectangular M-tensor is a rectangular S-tensor [17]. From Theorem 11 in [17], the following conclusion can be obtained easily.

Corollary 2 Let $\mathscr{A} \in \mathbb{R}^{[p ; q ; m ; n]}$ be a strong rectangular M-tensor. Then, the $\operatorname{RTCP}\left(\mathscr{A}, q_{m}, q_{n}\right)$ is feasible for all $q_{m} \in \mathbb{R}^{m}, q_{n} \in \mathbb{R}^{n}$.

\section{CONCLUSION}

In this paper, based on the definition of V-singular value for rectangular tensors, we extend elasticity M-tensors to rectangular M-tensors. Some properties of rectangular M-tensors are also presented. Finally, we prove that, an even-order partially symmetric rectangular $\mathrm{H}$-tensor with nonnegative diagonal entries is positive semidefinite and an even order partially symmetric rectangular H-tensor with positive diagonal entries is positive definite.

Acknowledgements: This work is supported by NSF of China (71461027, 11661084), Innovative talent team in Guizhou Province (Qian Ke He Pingtai Rencai [2016]5619), New academic talents and innovative exploration fostering project (Qian Ke He Pingtai Rencai [2017]5727-21), and Guizhou Province Natural Science Foundation in China (Qian Jiao He KY [2020]094).

\section{REFERENCES}

1. Chang K, Qi L, Zhou G (2010) Singular values of a real rectangular tensor. J Math Anal Appl 370, 284-294.

2. He J, Liu Y, Xu J, Liu G (2019) V-singular values of rectangular tensors and their applications. $J$ Inequal Appl 2019, ID 84.
3. Ling C, Nie J, Qi L, Ye Y (2009) Bi-quadratic optimization over unit spheres and semidefinite programming relaxations. SIAM J Optim 20, 1286-1310.

4. Qi L, Dai H, Han D (2009) Conditions for strong ellipticity and M-eigenvalues. Front Math China 4, 349-364.

5. Ding W, Liu J, Qi L, Yan H (2020) Elasticity M-tensors and the strong ellipticity condition. Appl Math Comput 373, ID 124982.

6. Ling C, Qi L (2013) $l^{k, s}$-singular values and spectral radius of rectangular tensors. Front Math China 8, 63-83.

7. Li C, Li Y, Kong X (2014) New eigenvalue inclusion sets for tensors. Numer Linear Algebra Appl 21, 39-50.

8. Li C, Wang F, Zhao J, Zhu Y, Li Y (2014) Criterions for the positive definiteness of real supersymmetric tensors. J Comput Appl Math 255, 1-14.

9. Qi L (2005) Eigenvalues of a real supersymmetric tensor. J Symb Comput 40, 1302-1324.

10. Ding W, Qi L, Wei Y (2013) M-tensors and nonsingular M-tensors. Linear Algebra Appl 439, 3264-3278.

11. Ding W, Wei Y (2016) Solving multi-linear systems with M-tensors. J Sci Comput 68, 689-715.

12. Zhang L, Qi L, Zhou G (2014) M-tensors and some applications. SIAM J Matrix Anal Appl 35, 437-452.

13. Mo C, Li C, Wang X, Wei Y (2019) Z-eigenvalues based structured tensors: $M_{z}$-tensors and strong $M_{z^{-}}$ tensors. Comput Appl Math 38, ID 175.

14. Kannan M, Shaked-Monderer N, Berman A (2015) Some properties of strong $\mathrm{H}$-tensors and general $\mathrm{H}$ tensors. Linear Algebra Appl 476, 42-55.

15. Yang Y, Yang Q (2011) Singular values of nonnegative rectangular tensors. Front Math China 6, 263-378.

16. Gu Y, Wu W (2019) Partially symmetric nonnegative rectangular tensors and copositive rectangular tensors. J Ind Manag Optim 15, 775-789.

17. Zeng Q, He J, Liu Y (2020) Structured rectangular tensors and rectangular tensor complementarity problems. Math Probl Eng 2020, ID 3897981. 